$37 \mid 2006$

Langue(s) et religion(s) : une relation complexe dans l'enseignement du français hors de France $x V l^{e}-x X^{e}$ siècle

\title{
L'enseignement du français à Izmir après 1839
}

Les Frères des Ecoles chrétiennes

\section{Hanife Güven}

\section{OpenEdition \\ Journals}

Édition électronique

URL : https://journals.openedition.org/dhfles/70

DOI : $10.4000 /$ dhfles.70

ISSN : 2221-4038

Éditeur

Société Internationale pour l'Histoire du Français Langue Étrangère ou Seconde

Édition imprimée

Date de publication : 1 décembre 2006

Pagination : 76-92

ISSN : 0992-7654

Référence électronique

Hanife Güven, «L'enseignement du français à Izmir après 1839 », Documents pour l'histoire du français langue étrangère ou seconde [En ligne], 37 | 2006, mis en ligne le 01 juillet 2009, consulté le 27 mai

2021. URL : http://journals.openedition.org/dhfles/70 ; DOI : https://doi.org/10.4000/dhfles.70 


\title{
L'enseignement du français à \\ Izmir après 1839 \\ Les Frères des Ecoles chrétiennes
}

\author{
Hanife GUVEN \\ Université de Dokuz Eylul Izmir
}

\begin{abstract}
L'année 1839 est un grand tournant pour l'Empire ottoman : La déclaration du firman (charte) de Tanzimat (réformes) ouvre une nouvelle ère semée de réformes politiques et administratives, inspirées par la philosophie des Lumières. Le firman de 1855 redéfinit les droits politiques et la liberté de scolarisation des communautés non musulmanes. Cette effervescence sociale est marquée par la prise de conscience de l'importance de l'éducation. L'introduction de la langue française sur le territoire ottoman se fait en grande partie par l'entremise des missionnaires catholiques. A Izmir c'est aux Frères des Ecoles chrétiennes que revient la tâche essentielle d'enraciner une tradition de l'enseignement de la langue française. Leur offre éducative de qualité attire les enfants des élites musulmanes qui auront leur rôle dans la transformation de la société ottomane.

The year 1839 is considered as a turning-point for the Ottoman Empire. The Declaration of the Act of Reform initiated a new era during which ideas derived from the Enlightenment were to be applied in both politics and administration. This was reaffirmed by the Act of 1855 , which would redefine the political rights and freedom of education for the non-muslim communities. The introduction of the French language in the lands of the Ottoman Empire was generally achieved through Catholic missionaries. In Izmir, the tradition of teaching French was carried out by the Frères des Ecoles chrétiennes. They offer a quality education to the children of the Ottoman elite, who would play an active role in both the growth and transformation of the Ottoman society.
\end{abstract}


L'année 1839 marque un grand tournant pour l'Empire ottoman: La déclaration du firman (charte) de Tanzimat (réformes) le 3 novembre 1839 ouvre une nouvelle ère semée de réformes politiques et administratives inspirées par la philosophie des Lumières. C'est l'expression par l'Etat même d'une volonté d'ouverture et de réforme. Elle est réaffirmée par le firman de 1855 qui va redéfinir les droits politiques et la liberté de scolarisation des communautés non musulmanes. Cette effervescence sociale est marquée par la prise de conscience de l'importance de l'éducation. Vers la fin du siècle, les écoles notamment les écoles privées vivront leur âge d'or en augmentant aussi bien en quantité qu'en qualité.

Sur le plan social, tout projet de modernisation se focalise sur les enjeux identitaires qui oscillent entre d'un côté la volonté de maintenir les traditions islamiques et de l'autre celle d'européaniser la société en en empruntant les valeurs, les lois et les structures institutionnelles. La querelle entre les deux camps se concrétise dans la personnalité des élites administratives, certains défendent ardemment la laïcisation et la démocratisation alors que pour d'autres les sources islamiques sont les bases incontournables de l'Etat et de la société. Le modèle civilisateur étant la France, l'un des moteurs les plus puissants de la modernisation est donc la langue et la culture française.

L'introduction de la langue française sur le territoire ottoman se fait en grande partie par l'entremise des missionnaires catholiques qui sont pour la plupart d'origine française. A partir du $\mathrm{XVI}^{\mathrm{e}}$ siècle, se propagent dans l'Empire ottoman les missions de divers ordres (Jésuites, Franciscains, Capucins, Dominicains, Lazaristes etc.) dont l'objectif fondamental est d'accompagner les catholiques dans la vie quotidienne dans un pays étranger. A partir de 1830, l'ambiance d'ouverture et la volonté de modernisation de l'Empire ottoman contribuent à leur action autant que le soutien du Pape Grégoire XVI. A Izmir, c'est aux Frères des Ecoles chrétiennes que revient la tâche essentielle d'enraciner une tradition de l'enseignement de la langue française. Réservée en principe aux non musulmans au début, leur offre éducative se distingue tout de suite par sa qualité et attire également les enfants des élites musulmanes qui auront leur rôle dans la transformation de la société ottomane. En raison de l'ampleur du sujet, nous limiterons notre propos aux aspects 
les plus significatifs de l'action des Frères des Ecoles chrétiennes ${ }^{110}$ dans la ville d'Izmir ${ }^{111}$ après 1839 sur une période qui va jusqu'à la fin du siècle.

\section{Aperçu historique}

Des écoles étrangères existaient sur le territoire ottoman depuis les privilèges religieux accordés par le sultan Mehmet II aux Latins catholiques et aux Grecs orthodoxes. Par la suite, sur la demande des communautés non musulmanes auprès du Vatican, des prêtres avaient été envoyés pour subvenir aux besoins en éducation religieuse et civile de ces derniers. Le voyageur Tournefort (1718, p. 413-414) a remarqué l'action bénéfique des missionnaires de divers ordres présents en Asie Mineure au début du XVIII ${ }^{\mathrm{e}}$ siècle :

(..) on voit tous les jours en Levant de nouveaux Capucins, des Dominicains, des Carmes, des Jésuites, des Prêtres des Missions étrangères à Paris. Ils instruisent ceux qui se présentent, ils baptisent, ils ramènent au bercail les brebis égarées, ils ouvrent les portes du Ciel aux Elus.

Izmir qui se vante d'un passé très chrétien ${ }^{112}$ ne pouvait pas rester en marge de l'essor de la mission religieuse. On enregistre l'établissement des Capucins de Saint-Polycarpe dans la ville dès 1610. Mais l'introduction du français se fait par l'intermédiaire des Jésuites ${ }^{\mathrm{i} 13}$ dont

${ }^{110}$ Je remercie les Frères de la Maison Généralice des archives des Frères des Ecoles chrétiennes à Rome, plus particulièrement le Frère Alain Houry, de sa gentillesse désintéressée et de son intarissable énergie. Je remercie également Didier Laroche, responsable du Centre de Recherche Antoine Galland à Izmir, qui m'a transmis les écrits du Frère Ange Michel.

${ }^{111}$ Une monnaie frappée sous Aydinoğlu Mehmet Bey et trouvée dernièrement atteste que Smyrne, le nom ancien de la ville, a été remplacé par celui d'Izmir dès 1317 (Milliyet, 09-X-2007).

112 Dès les premières heures du christianisme, elle faisait partie des Sept Eglises fondées en Asie Mineure avec son évêque saint Polycarpe, martyr en 166. On trouve dans le maitre-autel de la cathédrale une pierre commémorative offerte par l'Eglise de Lyon à l'Eglise Mère de Smyrne.

${ }_{113}$ L'arrivée des premiers Jésuites à Constantinople remonte au 8 novembre 1583. En effet, sur la demande de la communauté catholique de Galata et de Pera, 
l'objectif principal au départ, est, comme on l'imagine, loin d'être linguistique mais plutôt culturel et éducatif. On lit le témoignage de l'archevêque grec à leur propos, dans une requête adressée à Louis XIII « très chretien roy de France et de Navarre » :

Depuis qu'ils se sont établis au diocèse de votre Saint archevêché de Smyrne, ils n'ont jamais cessé d'assister toute sorte de chrestiens, soit par le bon exemple de leur vie, soit par leur prédication en nostre église, soit en enseignant aux enfants de nostre rite toutes sortes de sciences, avec la crainte de Dieu et les bonnes mœurs (Arch. NH 616, NH 206).

Le premier établissement scolaire français à Izmir remonte à 1638 , il fonctionne jusqu'à ce qu'il soit détruit pendant le tremblement de terre de 1688 qui, accompagné d'un incendie, détruit les trois quarts de la ville et les archives de l'école. L'abolition par le pape de la Compagnie de Jésus, en 1773, oblige les Jésuites à passer le relais aux Lazaristes qui dès 1782 inaugurent "la grande œuvre de l'apostolat et les œuvres d'assistance en Orient au 19 $9^{\mathrm{e}}$ siècle » (NH616 Smyrne dos. 6).

Du côté ottoman, jusqu'en 1839, sauf quelques exceptions comme les écoles militaires - elles ne sont pas sans importance pour autant -, l'enseignement du français est limité aux cercles religieux en priorité pour les non musulmans, aux cours privés et aux bureaux de traduction dans les ministères pour les élites musulmanes. En 1847 est fondé un ministère de l'Education nationale dans le sens moderne, c'està-dire, qui prépare la voie à la laïcisation de l'éducation, en l'arrachant au monopole des ulema. La première Réglementation (Maarif- $i$ Umumiye Nizamnamesi) qui réorganise l'éducation dans son ensemble (niveaux, méthodes, contenus), ne date que de $1869^{114}$. Les

faite au pape par l'intermédiaire de l'ambassadeur de France Germigny et du baile vénitien, cinq missionnaires jésuites arrivent à Istanbul le 8 novembre 1583 et s'installent le 18 au monastère Saint-Benoît. Dès leur installation ils ouvrent une école ( $c f$. Polvan 1952, p. 78 et 799). Leur mission était aussi d'accompagner les orphelins et les malades et de catéchiser les prisonniers.

114 Elle instaure l'obligation de l'enseignement primaire et fixe les niveaux comme suit: les écoles sibyan (maternelle ou premier niveau de l'enseignement primaire) seront ouvertes dans les quartiers et les villages, les rüştiye (deuxième niveau de l'enseignement primaire) dans les sous-préfectures qui comptent plus de 500 familles, les idadiye (collèges) dans les sous-préfectures de plus de 500 familles, 
fonctionnaires sont en général autodidactes. De même que les voyageurs européens, les élites intellectuelles qui font des voyages en Europe sont assez critiques devant la situation lamentable du pays, résumée dans les vers de Ziya Paşa :

J'ai visité les pays des infidèles, j'ai vu des villes et des palais. / J'ai parcouru les pays de l'Islam, je n'ai vu que des ruines. Il se trouve que j'ai été, moi aussi, à l'asile de la Sublime Porte / J'y ai vu tant d'insensés qui n'appréciaient pas Platon ${ }^{115}$.

L'éducation générale dispensée dans les écoles paroissiales n'était pas d'une importance considérable, tant du point de vue du nombre des écoles que du point de vue de l'offre. Dire que «les enfants ne connaissent même pas leur pater » recouvrait une réalité (Ergin 1939, p. 637). Il est difficile de ne pas souscrire aux remarques suivantes attribuées au Frère Hilaire (Arch. NH 616, NH 206) :

\begin{abstract}
Avant l'arrivée des missionnaires français en Orient, ce pays croupissait dans l'ignorance et la misère la plus noire. Pour les enfants, point d'école ; car on ne peut pas donner ce nom ni aux médréssés où, avec le Coran, on se contentait d'apprendre aux jeunes Turcs le mépris et la haine du chrétien; ni aux écoles paroissiales des Grecs et des Arméniens, où l'enseignement élémentaire était aussi insuffisant que l'enseignement religieux lui-même (La France catholique en Orient).
\end{abstract}

\title{
L'action des Frères des Ecoles chrétiennes à Smyrne
}

Appelés aussi «Lassalliens », les Frères des Ecoles chrétiennes sont les disciples de Jean-Baptiste de La Salle (1631-1719), canonisé en 1900 et déclaré « le patron spécial de tous les éducateurs chrétiens » en 1950. L'Institut des Frères des Ecoles chrétiennes, fondé en 1680, «a pour fin

les sultaniye (lycées) dans les chefs-lieux de département et le Darülfünun (l'université) à Istanbul.

${ }^{115}$ Diyar-1 küfrü gezdim, beldeler, kâşaneler gördüm / Dolaştım mülkü Islâmı, bütün viraneler gördüm. Bulundum ben dahi dar-üş-şifa-yı Bab-1 Âli'de / Felatun'u beğenmez anda çok divaneler gördüm (Gazel 1870). 
l'éducation chrétienne et civile de la jeunesse, et spécialement des enfants des artisans et des pauvres » (prospectus 1841, art. 2).

Vers la fin de la première moitié du XIX ${ }^{\mathrm{e}}$ siècle, les Frères sont appelés dans des pays très divers : en Suisse (1839), aux Etats-Unis d'Amérique, en Turquie (1841), en Egypte (1847) et en Allemagne (1850) (Bedel 2003, p. 108-110). La Méditerranée orientale est l'aire principale d'action des Lassaliens à cette époque et Smyrne est la première ville du Levant à les accueillir. Elle sera suivie la même année par la capitale, Constantinople. On remarque, à l'époque, dans la ville, deux quartiers bien distincts : celui des Turcs et celui des Francs. Le voyageur Vimercati (1852) y verra "l'aspect d'une république fédérative». Selon le voyageur Eyriès (1839, p. 371) «l'esprit commercial y adoucit les rapports entre des hommes de mœurs et de croyances diverses ». Selon le Dizionario di erudizione storicoecclesiastica (1854, p. 122-129) de Gaetano Moroni Romano, on estime la population à 130000 habitants dont 23000 Grecs, 7000 Arméniens, 12000 Juifs, descendants de ceux expulsés d'Espagne et du Portugal, 3000 Européens, mais aussi des habitants d'origine tartare ou chinoise $^{116}$. Dans l'archevêché, on compte 12000 catholiques, 1000 Arméniens, plus de 600 Persans, ou encore des habitants originaires de Damas ou d'Alep.

L'arrivée des Frères dans la ville se fait sur la demande du supérieur général de la Congrégation de Saint Lazare. Le jeune archevêque latin de la ville de Smyrne, Antoine Mussabini, qui n'est pas au courant de la démarche pour avoir été en visite à Lyon, considère l'acte comme "mépris à l'autorité et à la dignité épiscopale » ${ }^{117}$. Un échange de correspondance de quelques mois permettra l'obtention du

${ }^{116}$ Les séismes et les incendies sont très fréquents à Smyrne, ce qui a pour conséquence que la population varie en nombre.

${ }^{117} C f$. la Lettre du 10 juin 1841, écrite depuis Lyon par l'Archevêque d'İzmir au Supérieur des Frères des Ecoles chrétiennes : « Monsieur le Supérieur Général, j’ai appris avec surprise qu'accédant à la demande de MM. les Lazaristes, vous avez envoyé à Smyrne quelques uns de vos frères pour se consacrer à l'instruction de la jeunesse du pays. Je connais trop Mr. Le Supérieur l'admirable institution à la tête de laquelle vous êtes placé, pour ne pas voir d'avance le bien qui résulterait des efforts de vos bons frères mais je ne puis m'empêcher de désapprouver hautement la démarche des Lazaristes qui, en mon absence de Smyrne et sans aucune autorisation de ma part, se sont permis d'appeler dans mon Diocèse une nouvelle corporation religieuse ». 
décret épiscopal daté du 13 novembre 1841. Il est convenu finalement que les cinq frères ${ }^{118}$ sont là non pour fonder une nouvelle maison religieuse mais pour aider les Lazaristes à faire comme simples collaborateurs la classe gratuitement. Dans le prospectus daté de 1841, sont soulignés les divers principes de fonctionnement comme la gratuité, la discipline, le nombre de frères (au moins au nombre de trois dans chaque Maison, dont deux pour diriger les classes et le troisième pour gérer le temporel, Art. 4), les rémunérations et l'habitation des Frères (parloir, cuisine, réfectoire, dortoir, chambre d'exercice, chapelle ou oratoire, infirmerie, cave, bûcher, grenier, cour, jardin, puits ou pompe; des classes contiguës à courant d'air, et bien éclairées (Art. 5).

L'instruction religieuse occupe le premier rang :

Ils feront entendre la sainte Messe à leurs écoliers tous les jours d'école, à moins que le trop grand froid, la pluie etc. ne le permettent pas. Les dimanches et fêtes, ils assisteront avec eux à la messe de paroisse et aux Vêpres, supposé qu'on leur assigne dans l'église une place convenable ; les mêmes jours ils leur feront le catéchisme, pendant une heure et demie, le tout suivant l'usage de leur Institut (article 11).

Les Frères doivent respecter rigoureusement les valeurs de l'institution pour maintenir son caractère uniforme et universel :

Ils [les Frères] feront leurs Ecoles selon la méthode simultanée, développée dans le livre de la Conduite des Ecoles chrétiennes ${ }^{19}$; on n'y pourra rien changer, non plus qu'à leurs règles et à leur régime, afin qu'ils puissent conserver l'uniformité, qu'ils regardent comme un des principaux soutiens de leur société.

Les branches de l'enseignement sont celles qui sont comprises dans le brevet de capacité du degré élémentaire auxquelles on ajoute quelques notions de dessin linéaire (article 12).

En 1845, un incendie dévore la ville et 3000 maisons sont brûlées; l'établissement des Frères subit le même sort, mais ces derniers sauvent le mobilier. Leur maison fut reconstruite grâce à l'aide de la Propagation

${ }^{118}$ Ce sont les Frères Stanislas-Kotska (50 ans), Urain (28 ans), Victrice (19 ans), Andrieux (25 ans), Pilingot (43 ans).

${ }^{119}$ Italiques dans le texte. 
de la foi sur le même emplacement. Comme on vient de le voir dans les relations des voyageurs de l'époque, Izmir est une ville cosmopolite et européenne où une dizaine de groupes ethniques vivent en harmonie. Mais cela n'empêche pas la concurrence et les conflits entre ces diverses communautés, grecque, arménienne, italienne, française, appelées millet (nation) et parfois même à l'intérieur des confréries de divers ordres, comme chez les Lazaristes et les Jésuites. Les passages suivants témoignent des tensions provoquées par la concurrence entre les écoles des diverses communautés :

Monsieur le Ministre, le Gouvernement italien fait, depuis quelque temps, de puissants efforts \& impose de sérieux sacrifices, pour répandre la langue italienne dans la ville de Smyrne \& augmenter par là son influence. Déjà deux établissements d'instruction reçoivent de lui des subventions assez importantes, à la condition d'enseigner l'italien et tout dernièrement son Consul Général s'est mis en mesure d'établir une Ecole nationale de garçons et de filles pour la direction de laquelle doivent arriver prochainement des professeurs et des religieuses (Lettre du Consul Général H. Pelissier, 12 janvier 1881, adressée au Ministre des Affaires Etrangères Arch. NH 616 NH 206).

L'amélioration que je vous ai signalée l'an dernier nous a mis bien à l'aise, et le nombre des élèves s'est accru; mais le succès de l'école nous a attiré les foudres de la "Nation Grecque ». En pleine église, défense a été faite aux parents de nous confier les enfants. La population pauvre et ignorante, s'est tout d'abord à peu près soumise, en se lamentant; mais déjà sa patience est à bout, et, dans un avenir assez rapproché, cette crise grecque se résoudra d'elle-même, à l'instar de toutes celles que nous sommes habitués à rencontrer partout, après l'ouverture de nos classes. Ici, comme ailleurs, il faut savoir attendre (Rapport du Frère Visiteur de Turquie à Mgr Charmetant, dir. Gén. $B E C, 6^{\mathrm{e}}$ année $\mathrm{n}^{\mathrm{o}} 1$, janvier 1912 , p. 92). 


\section{Le français gagne du terrain}

Avant l'arrivée des missionnaires français, les langues les plus parlées dans la ville après le turc sont le grec et l'italien, sous l'influence de l'activité commerciale de Venise et de Gênes avec Byzance. L'augmentation du nombre de locuteurs francophones doit beaucoup à l'effort des Frères. En 1850, lors de son passage à Izmir pendant son deuxième voyage en Orient, Alphonse de Lamartine (1863, p. 188-200) assiste à la distribution des prix dans le collège des Lazaristes et prononce un discours dans lequel il souligne la mission éducative des Frères :

C'est à cette vulgarisation des langues européennes ici et des langues asiatiques chez nous que se consacrent vos respectables maîtres les Lazaristes, que je louerais plus librement s'ils n'étaient pas présents! Ordre aussi politique que religieux, aussi national que pieux, qui n'agite pas le monde par le bruit de ses ambitions et de ses controverses, mais qui accomplit sans bruit et sans brigues son apostolat modeste de civilisation dans le Levant! Ordre vraiment tolérant, qui porte et qui inspire la paix et la concorde, au lieu des disputes et des controverses de dogmes qui séparent et qui aigrissent les cœurs et les opinions! (p. 192)

En 1853, la communauté s'émancipe de la tutelle des Lazaristes. Le nombre des élèves augmente continuellement; dès 1854, 600 élèves fréquentent l'institut et trois autres frères se joignent aux anciens. Pour augmenter les ressources, on crée un demi-pensionnat qui soulèvera encore une fois des difficultés avec l'archevêque qui s'y oppose. En 1863, le passage par Izmir du Sultan Abdul Aziz ${ }^{120}$ (1861-76) est marqué par une fête mémorable de trois jours. Le règne d'Abdul Aziz est une période de perpétuelle mutation avec la création d'institutions, l'augmentation du nombre d'écoles modernes et surtout le développement du journalisme qui commence à créer une opinion

${ }^{120}$ Fils du Sultan Mahmut II (1785-1839) et frère d'Abdülmecit (1823-1861), son prédécesseur; Abdülaziz (1830-1876) est célèbre pour ses voyages aussi bien à l'extérieur qu'à l'intérieur de la Turquie. A l'occasion de l'Exposition universelle, il a effectué un voyage en France entre les 21 juin et 7 août 1867. Destitué en 1876, il sera trouvé mort, quelques jours après, les veines coupées. 
publique dans la société. A Constantinople ${ }^{121}$, le Lycée Galatasaray, baptisé «fenêtre donnant sur l'Occident», sera inauguré en 1868 et assurera l'emprise de la langue et de la culture françaises jusqu'en 1914, date d'arrivée des Allemands. Le projet est unique en son genre en ce sens qu'il regroupe en son sein les élèves de diverses communautés et de diverses religions et offre un enseignement en français. L'administration et l'enseignement sont confiés aux enseignants français (Karal 1988, p. 203). Avec un effectif à moitié musulman, le lycée Galatasaray attirera non seulement la méfiance de la Papauté qui soutient d'habitude les actions catholiques, mais aussi celle des communautés orthodoxes et des Juifs ${ }^{122}$. Mais l'entreprise a réussi puisqu'on comptait 622 élèves ${ }^{123}$, un an après son inauguration, en décembre 1869 .

Le règne du sultan Abdulhamit II est marqué par l'installation des Frères dans les plus lointaines contrées de l'Asie mineure, de Trébizonde / Trabzon (1881) sur la Mer Noire, à Erzouroum / Erzurum (1883) à l'Est, en passant par Angora / Ankara (1892) au centre, sans compter les contrées qui ne font plus partie de la Turquie actuelle

${ }^{121}$ Constantinople, l'ancienne appellation de la ville après tant d'autres à l'époque ottomane (Konstantiniyye, Darü'l Mülk, Dersaadet), a été remplacée officiellement par Istanbul à partir de 1930.

${ }^{122}$ La religion étant la base de toute morale, imposer le même programme, la même alimentation, le même calendrier aux apprenants de divers rites religieux, les faire dormir dans les mêmes dortoirs, les faire vivre dans la même atmosphère n'est pas sans poser de problèmes. La crainte de l'influence que peut avoir la confession des enseignants joue un rôle déterminant dans l'engagement des parents (Sungu 1943 , p. 335-337). Par deux fois, la papauté défend aux catholiques orientaux de fréquenter l'école. Selon De Salve «Les catholiques auraient peur que la coexistence des ethnies différentes influence la morale des enfants catholiques » (Ergin 1939, p. 604-605). L'ambassadeur russe, le général Ignatyef, qui ne cache pas son mécontentement devant l'influence française dans la capitale ottomane grâce à une telle école, incite à ne pas fréquenter celle-ci ; les Grecs orthodoxes se plaignent que leur langue occupe peu de place. Les Juifs, méfiants eux aussi, stipulent qu'on respecte leur foi quant à l'alimentation de leurs enfants. La presse ottomane, au contraire, encourage les parents en leur asssurant que les ingénieurs, enseignants, économistes du futur seront formés dans cette école (Engelhardt 1999, p. 250-251, Berkes 1978, p. 239).

123622 élèves dont 277 Musulmans, 91 Arméniens, 85 Grecs, 65 Latins catholiques, 40 Bulgares, 29 Juifs, 28 Arméniens catholiques, 7 Protestants (Engelhardt 1999, p. 483). Il y en avait 341 à l'inauguration le $1^{\mathrm{e}}$ septembre 1868 , dont 147 Musulmans, 48 Arméniens grégoriens, 36 Grecs, 34 Juifs, 34 Bulgares, 23 Latins catholiques, 19 Arméniens catholiques (Sungu 1943, p. 335-336 ). 
comme Thessalonique (1888), Canée (1901), et Chios (1902) (Bédel 2003, p. 104).

\section{Le modèle français d'éducation}

Les Frères d'Izmir franchissent en 1872 une nouvelle étape avec l'ouverture du cycle secondaire auquel s'ajoute un pensionnat pour augmenter leurs ressources. A partir de 1880, une ère de prospérité et d'essor commence pour ces derniers, malgré «l'hostilité du Gouvernement français à l'égard des Congrégations religieuses, et spécialement de celles qui se vouent à l'enseignement " (BEC 1923, p. 334). La même année, avec la bénédiction de l'archevêque A.P. Timoni, le nouveau local est inauguré rue de la Rose. Il est intéressant de parcourir le prospectus du collège Saint Joseph dirigé par les Frères des Ecoles chrétiennes, daté du 18 septembre 1880, pour constater le souci non seulement d'assurer une formation intellectuelle mais également de répondre à un besoin immédiat de la vie réelle. Les objectifs retenus sont définis en ces termes :

La sympathique confiance que nous témoigne depuis quarante ans l'honorable population de Smyrne nous fait un devoir de répondre aux désirs si souvent exprimés par beaucoup de pères de famille de voir compléter notre enseignement, en lui donnant tout le développement que réclament les besoins actuels de la société.

Le décret du 15 novembre 1811 limitait l'enseignement donné dans les écoles à la lecture, à l'écriture et au calcul (Bedel 2003, p. 65). Dans le collège, la religion constitue la base de l'éducation et de l'instruction, mais il s'assigne désormais la double tâche de permettre l'accès à la langue et à la culture françaises et d'assurer une formation aussi complète que possible «pour les enfants dont la position exige une éducation plus soignée et plus complète que celle donnée dans les écoles primaires ». Le programme est basé sur le modèle français dressé par le Ministère de l'Instruction Publique. La discipline " ferme et paternelle » est basée sur la confiance mutuelle. L'enseignement comprend les matières suivantes : 


\section{L'INSTRUCTION RELIGIEUSE}

2. LA LECTURE

3. LA CALLIGRAPHIE

4. LA LANGUE FRANÇAISE :

Grammaire, Orthographe, Analyse Grammaticale, id. Logique, Style épistolaire, Correspondance Commerciale, Narration, Description, Discours, Rhétorique, Eléments de logique.

5. LES LANGUES VIVANTES (1) :

Grec, Anglais, Italien, Turc, Allemand.

6. LES MATHEMATIQUES :

Arithmétique, Algèbre, Arpentage, Géométrie, Trigonométrie.

7. LA COMPTABILITE COMMERCIALE :

Tenue des livres, Comptes en participation, Cours de change, Calcul de banque, etc.

\section{LA GEOGRAPHIE :}

Physique, Politique, Commerciale, Historique.

\section{LA COSMOGRAPHIE}

10. L'HISTOIRE :

Sainte, Ancienne, Romaine, du Moyen Age, Moderne.

11. LES SCIENCES NATURELLES :

12. La Physique, la Chimie, et l'Histoire Naturelle.

13. LE DESSIN :

Linéaire, d'Architecture, de Perspective, de Projections, de Figure, d'Ornement.

14. LA MUSIQUE :

Vocale, Piano, Violon (2)

[(1) L'italien, le turc et l'allemand sont à la charge des parents. (2) Le piano et le violon sont à la charge des parents]. 
Suivant la politique linguistique de la France «toute l'instruction est donnée en français ». Quant aux enseignants, il est précisé, seulement pour les professeurs de langues, qu'ils sont « dignes de la confiance des familles ». En effet, l'offre linguistique frappe vivement par sa richesse et allait servir de modèle aux décideurs en matière de politique linguistique.

En 1881, un nouveau collège (Saint-André) ouvre ses portes dans un quartier appelé la Pointe (aujourd'hui Alsancak) peuplé de Grecs. En 1888, l'année de la béatification du fondateur Jean-Baptiste de La Salle, à la demande du curé de Cordélio (aujourd'hui Karşıyaka), commence la préparation de l'ouverture dans ce même quartier d'une autre école qui commencera l'enseignement l'année suivante.

Les élèves sont issus de la bourgeoisie ou de l'aristocratie des communautés chrétiennes, grecques, arméniennes et juives ${ }^{124}$. Les musulmans sont les derniers à s'adresser au collège, non sans causer quelque désarroi. La méfiance prévaut des deux côtés. Il est facile de deviner que les débouchés sont nombreux pour une formation aussi rentable ${ }^{125}$.

En 1899, dans l'archidiocèse de Smyrne on compte 6 écoles, 22 classes et 827 élèves répartis par cultes comme suit : 670 catholiques, 148 schismatiques (Grecs et Arméniens), 4 musulmans et 5 israélites (BEC 1923, p. 334). Dans un rapport adressé en 1901 à l'ambassadeur de France auprès de la Sublime Porte, on lit :

Nous avons à Smyrne 4 écoles bien fréquentées: une école d'enseignement secondaire moderne avec 230 élèves, deux grandes écoles gratuites comptant près de 450 enfants et une

${ }^{124}$ Ergin (1939, p 648) donne, pour l'année 1914, une liste où on peut voir la situation sociale des familles des élèves du collège Saint-Joseph d'Istanbul, tenu également par les Frères des Ecoles chrétiennes : princes (8), maréchaux et généraux (22), hauts dignitaires (90), directeurs de banques (30), fonctionnaires civils (80), banquiers et commissionnaires (80), médecins (90), avocats (20), ingénieurs (20), grands propriétaires (300).

${ }^{125}$ Les premiers musulmans à entrer dans ces écoles sont les huit enfants du Fuat Paşa qui avait dépensé toute son énergie pour les inscrire au collège Saint-Joseph de Kadıköy. Deux perdent la vie dans les guerres, les autres travaillent dans l'agriculture, le commerce, l'interprétariat et la traduction, la médecine et la politique. Aussi bien pour les services qu'ils ont rendus que par le chemin qu'ils ont parcouru, les enfants de Fuat Paşa ont démontré que ces écoles n'étaient pas des institutions dangereuses. 
école professionnelle d'art et métiers gratuite ayant 35 apprenants. Outre ces deux écoles, nous en avons deux dans la banlieue : A Cordelio et à Bournobat. Ces écoles réunissent près de 180 élèves. Il resterait à créer, Excellence, pour compléter nos moyens d'actions, une bonne école de 2 ou 3 classes à Geuz-Tepe, villégiature pleine d'avenir aux portes de Smyrne... Nous entourons Smyrne d'écoles françaises capables de conserver et d'étendre notre influence pour la diffusion de notre belle langue » (NH 616 Smyrne Dos. 7).

On constate deux points de vue contradictoires à propos des écoles tenues par les non musulmans. Ceux qui les connaissent de près, pour y avoir étudié par exemple, reconnaissent la qualité de l'offre éducative. Par contre, ceux qui ne les connaissent pas tombent dans le piège des généralisations rapides. Pour citer un exemple précis, il était devenu un lieu commun de distinguer pour l'époque de Tanzimat trois types d'écoles, chacune ayant sa particularité. Les écoles des petits (du quartier et du village) qui dispensaient une éducation générale plutôt religieuse, dépendaient du Ministère des Fondations; elles ont été suivies, à l'époque de la modernisation, sans être vraiment réformées, par des établissements du primaire et des collèges qui dépendaient du ministère de l'Education. Enfin, les écoles primaires ou collèges qui étaient destinés aux enfants non musulmans, étaient sous l'influence respective des ambassades et des patriarcats. Et de stigmatiser leurs objectifs respectifs en ces termes :

les premiers étaient sous l'influence des médressés, les seconds s'efforçaient de s'orienter vers l'Occident, les troisièmes visaient à démembrer les Ottomans (Koçer 1970, p. 82).

Dans un empire déclaré « malade ${ }^{126} »$ dès 1853 , exposés à toutes sortes d'épreuves et aux caprices du contexte géopolitique, les Frères doivent faire face efficacement à des situations et à des événements aussi inattendus que décourageants, tantôt en concurrence avec d'autres congrégations, tantôt aux prises avec des conflits internationaux, sans

${ }^{126} C f$. la phrase historique du tzar Nicolas $\mathrm{I}^{\text {er }}$, adressée en 1853 à sir Hamilton Seymour, ambassadeur anglais: "Nous avons dans nos bras (...) un homme gravement malade; ce serait, je vous le dis franchement, un grand malheur si, l'un de ces jours il mourait, surtout avant que toutes les dispositions soient prises (Dumont 1999, p. 538). 
compter les catastrophes naturelles. Durant la guerre de Crimée (18531856), le rapprochement franco-turc leur fait gagner du prestige ${ }^{127}$ auprès des autochtones, alors que durant la Première Guerre Mondiale, malgré un effectif qui dépasse le nombre de mille, ils seront obligés de quitter les lieux en dépit des efforts du gouverneur Rahmi Bey ${ }^{128}$.

\section{Conclusion}

L'ouverture culturelle et l'effervescence sociale créées vers la deuxième moitié du XIX ${ }^{\mathrm{e}}$ siècle sur le territoire ottoman d'une part et les initiatives du pape Grégoire XVI de l'autre favorisent énormément l'enracinement du français sur le territoire ottoman, aussi bien par l'augmentation des écoles des diverses congrégations que par son introduction progressive dans les curricula des écoles publiques.

L'efficacité de ce rôle instrumental est indissociable de la figure du Frère, voué aux sacrifices de toutes sortes, disposé à toutes les souffrances, agissant par obéissance et représentant sur un territoire étranger, au départ la religion catholique, mais par la suite aussi la langue et la nation françaises. Les archives des Frères des Ecoles chrétiennes à Rome nous montrent combien ils sont les représentants par excellence du colonialisme français, en ce sens qu'ils considèrent chaque nouvel apprenant, chaque nouveau bâtiment, chaque nouvelle école, comme une nouvelle conquête. Leur foi et leur persévérance à toute épreuve leur permettent de renaitre de leurs cendres avec le même enthousiasme. Leur tradition éducative se poursuit encore de nos jours

127 « La France et l'Angleterre se rangent aux côtés d'Abdulmedjid. Leurs flottes passent de l'archipel dans la mer Noire. Leurs troupes décident l'ennemi à s'éloigner du territoire ottoman ; puis, elles se transportent en Crimée. Du 9 octobre 1854 au 8 septembre 1855, ce sera le long siège de Sébastopol. Enfin, la paix est signée. Le Congrès de Paris a garanti l'intégrité de l'empire ottoman; et la France victorieuse jouit chez ses alliés, chez ses protégés, d'un nouveau prestige " (Rigault 1948, p. 361).

${ }^{128}$ Rahmi Bey (1873-1947), homme d'Etat ottoman anglophile, fut gouverneur d'Izmir entre le 19 septembre 1913 et le 24 octobre 1918 ; il est connu aussi pour sa politique et son administration libérales, et surtout pour sa position contre la déportation des Grecs et des Arméniens de la région, pendant la Première Guerre Mondiale (Mehmetefendioğlu 1993, p. 360). 
dans des écoles laïques privées bilingues avec le traditionnel prestige et le dynamisme de cette institution.

\section{BIBLIOGRAPHIE}

Documents Régionaux et Locaux : Turquie Smyrne. NH 616, NH 206 Archivio Fratelli delle Scuole Cristiane Casa Generalizia Roma.

BEDEL, H. 2003. Initiation à l'histoire de l'Institut des Frères des Ecoles chrétiennes, $\mathrm{XIX}^{\mathrm{e}}$ siècle, 1805-1875. Etudes lassalliennes 9. Rome : Maison Généralice FSC.

BERKES, N. 1978. Türkiye'de Çağdaşlasma, Doğu-Batı Yay. Istanbul.

«Le collège de Saint-Joseph, à Smyrne», Bulletin des Ecoles chrétiennes, Maison Saint-Joseph, Lembecq Lez Hal, $11^{\mathrm{e}}$ année, $\mathrm{n}^{\mathrm{o}} 1$, janvier 1923, p. 321-340.

DUMONT, P. 1999. «Il periodo dei Tanzimat » in Storia dell'Impero ottomano, p. 495-561.

ENGELHARDT. 1999. Tanzimat ve Türkiye, (Trad. Ali Reşad), Istanbul : Kaknüs.

ERGIN, O. 1939. Türkiye Maarif Tarihi, 1-2, Istanbul: Osmanbey Matbaas1.

EYRIES J-B. MDCCCXXXIX. Voyage pittoresque en Asie et en Afrique, Paris : chez Furne et Cie.

KOÇER, H.A. 1970. Türkiye'de Modern Eğitimin Doğuşu ve Gelişimi, Istanbul : MEB.

LAMARTINE, A. de. MDCCCLXIII. Nouveau voyage en Orient (1850), Paris : chez l'auteur.

MEHMETEFENDIOĞLU, A. 1993. «Rahmi Bey’in İzmir Valiliği» Çağdaş Türkiye Tarihi Araştırmaları Dergisi, 1 n 3, p. 347-370.

RIGAULT, G. 1948. Histoire Générale de l'Institut des Frères des Ecoles chrétiennes. t. IV. Paris : Plon.

SUNGU, I. 1943 «Galatasaray Lisesinin Kuruluşu» Belleten, VII, $\mathrm{n}^{\mathrm{o}} 28$, Ankara: TTK, p. 315-347.

TOURNEFORT, J. P. de. MDCCXVII. Relation d'un voyage du Levant, fait par ordre du roy, t. 2. Paris : Imprimerie Royale. 
«Turquie », Bulletin des Ecoles chrétiennes, Maison Saint-Joseph, Lembecq Lez Hal, $6^{\mathrm{e}}$ année, $\mathrm{n}^{\mathrm{0}} 1$, janvier 1912, p. 91-93. 